\title{
A Two-Stage Model Queueing with No Waiting Line between Channels
}

\author{
Vedat Sağlam ${ }^{1}$ and Müjgan Zobu' ${ }^{2}$ \\ ${ }^{1}$ Department of Statistics, Ondokuz Mayıs University, 55139 Samsun, Turkey \\ ${ }^{2}$ Department of Statistics, Amasya University, 05100 Amasya, Turkey \\ Correspondence should be addressed to Müjgan Zobu; mujganzobu@hotmail.com
}

Received 5 December 2012; Revised 22 April 2013; Accepted 20 May 2013

Academic Editor: Suiyang Khoo

Copyright ( 2013 V. Sağlam and M. Zobu. This is an open access article distributed under the Creative Commons Attribution License, which permits unrestricted use, distribution, and reproduction in any medium, provided the original work is properly cited.

We consider a new queuing model with sequential two stations (stages), single server at each station, where no queue is allowed at station 2 and with no restriction at station 1 . There is a FCFS service discipline in which the input stream is Poisson having rate $\lambda$. The service time of any customer at server $i(i=1,2)$ is exponential with parameter $\mu_{i}$. The state probabilities and loss probability of this model are given. The performance measures are obtained and optimized, and, additionally, the model is simulated. The simulation results, exact results, and optimal results of the performance measures are numerically computed for different parameters.

\section{Introduction}

New models of queuing theory have been needed lately concerning the developments in areas such as production line, communication, and computer systems. One of these necessary models is for a tandem queuing system. Many important studies have been done in this area. The mean waiting time and the mean customer number at two tandem channels (servers) which are Poisson arrival and exponential service time were given in [1]. The mean customer number, distribution of waiting time, and the probabilities of various numbers of the tandem queuing system at every stage of the Poisson arrival and the exponential service time of the tandem queuing system were found in [2]. In [3], it was proved that if arrivals to the system are Poisson process with the parameter, then the output of this system is also Poisson process with a parameter $\lambda$. A more complicated example with network analysis was studied in [4]. In queuing theory, it is usually assumed that service channels are homogeneous. However, it is seen that in real queuing systems, service channels sometimes are heterogeneous. Understanding such systems is important for finding solutions to both theoretical and technical problems. Under the condition that the sum of service rates is fixed, homogeneous systems have been compared with the heterogeneous systems for performance measures in [5-9]. The measures of effectiveness for tandem queues with blocking were calculated according to an approximation method and simulated in [10]. The tandem queues with one server in the first queue, and $n \geq 1$ servers in the second queue, where the arrivals to the system with Poisson process having parameter $\lambda$ and there is no waiting room between the two stages, were analyzed and some probabilities for the number of customers were found in [11]. In the literature usually, for some similar models to ours, blocked tandem queueing systems have been studied and probabilities of number of customers have been obtained. Approximate and simulation results of performance measures have been obtained. In our proposed model, there is no restriction for the first stage, there is no waiting room between both stages and no blocking (i.e., customers leave the system after having service in the first stage if the second stage is busy). Probabilities of being-nonbeing of customers in the first and second stages, performance measures, and the optimal values of these measures are theoretically obtained by analyzing our proposed method. Also, we have compared some results by obtaining simulation results. 
In real life, while there is waiting case in the service systems, because of obligations and urgency and unavailability of desired features, the loss may occur in the beginning of second stage as in our proposed model. Therefore, we have decided to construct such a model and analyze it. The following two examples can be proposed as potential applications of our model under some conditions.

The following examples can be proposed as the applications of our model on some topics.

(a) Fruit-Vegetable Packing Line. There is a company which exports fresh fruits and vegetables. This company has a product packing line which consists of two stages, for a special export fruit. The product comes to first stage for quality control. Later, if the product is not in the desirable size or quality, then it is taken away from the system (the loss occurs). The product is sent to the second stage to be packed with no waiting time, if it qualifies the desirable size and quality.

(b) VoIP (Voice Over Internet Protocol). Internet telephony refers to communications services-voice, fax, SMS, and/or voice-messaging applications - that are transported via an IP network, rather than the public switched telephone network (PSTN). The steps involved in originating a VoIP telephone call are signalling and media channel setup, digitization of the analogy voice signal, encoding, packetization, and transmission as Internet Protocol (IP) packets over a packet-switched network. On the receiving side, similar steps (usually in the reverse order) such as reception of the IP packets, decoding of the packets, and digital-to-analogy conversion reproduce the original voice stream.

More generally, the server transfers voice messaging to the recipient. In this system, the server is first service, the recipient is second service. If the recipient is busy, and then the server destroys voice messaging at that moment.

\section{The Model}

Let $n_{1}$ and $n_{2}$ be the number of customers in the first and second stages, respectively, at any time of $t$, including those being served, where $n_{1}=0,1,2, \ldots ; n_{2}=0,1$.

For stage $i$, let $\xi_{i}(t)$ be defined as follows:

$$
\xi_{i}(t)= \begin{cases}n_{1}, & i=1 \\ n_{2}, & i=2\end{cases}
$$

We can denote

$$
P_{n_{1}, n_{2}}(t)=\operatorname{Prob}\left\{\xi_{1}(t)=n_{1}, \xi_{2}(t)=n_{2}\right\} .
$$

The random process

$$
\left\{\xi_{i}(t): i=1,2 ; t \geq 0\right\}
$$

is a continuous-time two-dimensional Markov chain. For $n \geq$ 1 , the state space of this chain becomes

$$
E=\{(0,0),(0,1),(n, 0),(n, 0)\} .
$$

We wish to find the steady-state probability $p_{n_{1}, n_{2}}$ :

$$
P_{n_{1}, n_{2}}=\lim _{t \rightarrow \infty} P_{n_{1}, n_{2}}(t)
$$

The usual procedure leads to the steady-state equations for this Markov chain:

$$
\begin{gathered}
0=-\lambda p_{00}+\mu_{2} p_{10}, \\
0=-\left(\lambda+\mu_{2}\right) p_{01}+\mu_{1} p_{10}+\mu_{1} p_{11}, \\
0=-\left(\lambda+\mu_{1}\right) p_{n_{1}, 0}+\lambda p_{n_{1}-1,0}+\mu_{2} p_{n_{1}, 1}, \quad n_{1} \geq 1, \\
0=-\left(\lambda+\mu_{1}+\mu_{2}\right) p_{n_{1}, 1}+\lambda p_{n_{1}-1,1} \\
+\mu_{1}\left(p_{n_{1}+1,0}+p_{n_{1}+1,0}\right), \quad n_{1} \geq 1 .
\end{gathered}
$$

We define $\rho_{i}=\lambda / \mu_{i}$ for $i=1,2$ and $\rho=\lambda /\left(\mu_{1}+\mu_{2}\right)$.

2.1. State Probabilities. The probability $p_{n_{1}}$ denotes the probability of finding $n_{1}$ customers in the first stage at an arbitrary point in time (see [12]). We can write these as

$$
\begin{gathered}
p_{n_{1}}=p_{n_{1}, 0}+p_{n_{1}, 1}=\rho_{1}^{n_{1}}\left(1-\rho_{1}\right), \quad n_{1} \geq 0, \\
p_{00}+p_{01}=1-\rho_{1} .
\end{gathered}
$$

Using this equation and (6), we obtain the following:

$$
\begin{gathered}
p_{01}=\frac{\lambda\left(1-\rho_{1}\right)}{\lambda+\mu_{2}}=\frac{\left(1-\rho_{1}\right) \rho_{2}}{1+\rho_{2}}, \\
p_{00}=\frac{\mu_{2}}{\lambda} p_{01}=\frac{1-\rho_{1}}{1+\rho_{2}} .
\end{gathered}
$$

By substituting the expression $p_{n_{1}+1}$ in (9), we get

$$
\left(\lambda+\mu_{1}+\mu_{2}\right) p_{n_{1}, 1}=\lambda p_{n_{1}-1,1}+\mu_{1} p_{n_{1}+1}, \quad n_{1} \geq 1 .
$$

Let us take $a=\rho /(1+\rho)$ and choose $p_{n_{1}, 1}$ a place to put $y_{n_{1}}$ in (13), for simplicity. In this case, the following equation can be obtained:

$$
y_{n_{1}}=a y_{n_{1}-1}+a y_{n_{1}}, \quad n_{1} \geq 1
$$

Both sides of (14) are divided into $a^{n_{1}}$, and later, the index $n_{1}$ is changed to $k$. Then, we sum this obtained value:

$$
\begin{gathered}
\frac{y_{n_{1}}}{a^{n_{1}}}-y_{0}=\sum_{k=1}^{n_{1}} \frac{p_{k}}{a^{k-1}}=\frac{\rho_{1}\left(1-\rho_{1}\right)\left(a^{n_{1}}-\rho_{1}^{n_{1}}\right)}{\left(1-\left(\rho_{1} / a\right)\right) a^{n_{1}}}, \\
p_{n_{1}, 1}-a^{n_{1}} p_{01}=a \frac{\rho_{1}\left(1-\rho_{1}\right)\left(a^{n_{1}}-\rho_{1}^{n_{1}}\right)}{a-\rho_{1}}, \\
p_{n_{1}, 1}=a \frac{\rho_{1}\left(1-\rho_{1}\right)\left(a^{n_{1}}-\rho_{1}^{n_{1}}\right)}{a-\rho_{1}}+\frac{\left(1-\rho_{1}\right) \rho_{2}}{1+\rho_{2}} a^{n_{1}} .
\end{gathered}
$$


When the value of $a$ in (17) is substituted, the following equation is obtained:

$$
p_{n_{1}, 1}=\frac{\left(1-\rho_{1}\right) \rho_{2}}{1+\rho_{2}} \rho_{1}^{n_{1}} \text {. }
$$

We get the probability $p_{n_{1}, 0}$ from (10) and (18):

$$
p_{n_{1}, 0}=\frac{\left(1-\rho_{1}\right)}{1+\rho_{2}} \rho_{1}^{n_{1}} .
$$

2.2. Loss Probability. The customer's loss probability is given as

$$
p_{L}=\sum_{n_{1}=0}^{\infty} p_{n_{1}, 1}=\frac{\rho_{2}}{1+\rho_{2}} .
$$

In other way, the formula (20) can be obtained from "Erlang's loss formula" or "Erlang's B formula" for the $M / M / c / c$ queue. In [13], it is denoted as $B\left(c, \rho_{2}\right)$ and formulated as the follows:

$$
B\left(c, \rho_{2}\right)=\frac{\rho_{2} / c !}{\sum_{n=0}^{c} \rho_{2}^{n} / n !},
$$

where $\rho_{2}$ is the utilization factor. Substituting $c=1$ in (21), we have (20).

\section{The Measures of Performance and Optimization of Performance Measures}

3.1. The Mean Sojourn Time. Let $T$ be a random variable that describes the sojourn time of customers in the system. Using the law of total expectation, we can write is as follows

$$
E(T)=E(T \mid A) P(A)+E(T \mid \bar{A}) P(\bar{A}),
$$

where $P(A)$ is the probability of the loss of a customer. Now it is clear that

$$
E(T \mid A)=\frac{1}{\mu_{1}-\lambda}, \quad E(T \mid \bar{A})=\frac{1}{\mu_{1}-\lambda}+\frac{1}{\mu_{2}} .
$$

Thus,

$$
E(T)=\frac{\mu_{1}+\mu_{2}}{\left(\mu_{1}-\lambda\right)\left(\mu_{2}+\lambda\right)} .
$$

Our main results about the problem of minimizing the mean sojourn time can be explained by the following theorem.

Theorem 1. If sum of two service rates $\mu_{1}+\mu_{2}=\mu$ is fixed, then the mean sojourn time of this tandem system attains its minimum value for $\mu_{1}=\mu / 2+\lambda$ and $\mu_{2}=\mu / 2-\lambda$.
Proof. We will prove the theorem by using the following inequality:

$$
\left(\prod_{\dot{\mathrm{I}}=1}^{m} a_{i}\right)^{1 / m} \leq \frac{1}{m} \sum_{i=1}^{m} a_{i}, \quad \forall a_{i}>0, \forall m \in \mathbb{Z}^{+} .
$$

From inequality (25), we have

$$
\frac{1}{\left(\mu_{1}-\lambda\right)\left(\mu_{2}+\lambda\right)} \geq \frac{4}{\mu^{2}} .
$$

If we replace the expressions $\mu_{1}+\mu_{2}=\mu$ and $4 / \mu^{2}$ in equality (24), we obtain the minimum value of $E(T)$ as follows:

$$
\min E(T)=\frac{4}{\mu},
$$

where the equality (27) is provided with $\mu_{1}=\mu / 2+\lambda$ and $\mu_{2}=\mu / 2-\lambda$.

3.2. The Mean Number of Customers. Let $N$ be the random variable that describes the number of customers in the system:

$$
\begin{aligned}
E(N) & =\sum_{n_{1}=0}^{\infty} \sum_{n_{2}=0}^{1}\left(n_{1}+n_{2}\right) p_{n_{1}, n_{2}} \\
& =\frac{\lambda\left(\mu_{1}+\mu_{2}\right)}{\left(\mu_{1}-\lambda\right)\left(\mu_{2}+\lambda\right)},
\end{aligned}
$$

or

$$
E(N)=\lambda E(T)
$$

The mean number of customers in the system is optimized from Theorem 1 and the equality (28b) as below:

$$
\min E(N)=\lambda \min E(T)=\frac{4 \lambda}{\mu} .
$$

The independence of the number of customers can be expressed by the following theorem.

Theorem 2. If the random variables $N_{1}$ and $N_{2}$ are taken as the number of customers in the first and second stages, respectively, then $N_{1}$ and $N_{2}$ are independent random variables.

Proof. The joint probability mass functions of $N_{1}$ and $N_{2}$ random variables is

$$
p_{n_{1}, n_{2}}=P\left(N_{1}=n_{1}, N_{2}=n_{2}\right), \quad n_{1}=0,1,2, \ldots ; n_{2}=0,1 .
$$

If $n_{2}=0$ and $\left(n_{2}=1\right)$ are substituted in (30), the equations (19) and (18) are obtained, respectively.

The marginal probability mass function of $N_{1}$ is given as (10) 
TABLE 1: For $\lambda=0.30, \mu_{1}=0.80 ; \mu_{2}=1.00$.

\begin{tabular}{lcccccc}
\hline $\begin{array}{l}\text { Iteration } \\
\text { number }\end{array}$ & \multicolumn{2}{c}{ Simulation results } & \multicolumn{2}{c}{ Exact results } & \multicolumn{2}{c}{ Optimal results } \\
& $E(T)$ & $E(N)$ & $E(T)$ & 2.7692 & 0.8308 & 2.2222 \\
100 & 2.8163 & 0.8452 & 2.7692 & 0.8308 & 2.2222 \\
1000 & 2.8111 & 0.8433 & 2.7692 & 0.8308 & 2.2222 & 0.6667 \\
5000 & 2.8124 & 0.8436 & 0.6667 \\
\hline
\end{tabular}

TABLE 2: For $\lambda=0.1, \mu_{1}=0.9 ; \mu_{2}=0.9$.

\begin{tabular}{lcccccc}
\hline $\begin{array}{l}\text { Iteration } \\
\text { number }\end{array}$ & \multicolumn{2}{c}{ Simulation results } & \multicolumn{2}{c}{ Exact results } & \multicolumn{2}{c}{ Optimal results } \\
& $E(T)$ & $E(N)$ & $E(T)$ & 2.2500 & 0.2250 & 2.2220 \\
100 & 2.2661 & 0.2263 & 2.2500 & 0.2250 & 2.2220 & 0.2222 \\
1000 & 2.2599 & 0.2261 & 2.2500 & 0.2250 & 2.2220 & 0.2222 \\
5000 & 2.2500 & 0.2260 & 0.2222 \\
\hline
\end{tabular}

TABLE 3: For $\lambda=0.01, \mu_{1}=0.6 ; \mu_{2}=0.7$.

\begin{tabular}{lcccccc}
\hline $\begin{array}{l}\text { Iteration } \\
\text { number }\end{array}$ & \multicolumn{2}{c}{ Simulation results } & \multicolumn{2}{c}{ Exact results } & \multicolumn{2}{c}{ Optimal results } \\
& $E(T)$ & $E(N)$ & $E(T)$ & 3.1034 & 0.0310 & 3.0769 \\
100 & 3.1042 & 0.0311 & 3.1034 & 0.0310 & 3.0769 \\
1000 & 3.1034 & 0.0310 & 3.1034 & 0.0310 & 3.0769 \\
5000 & 3.1043 & 0.0310 & $T(N)$ & 0.0308 \\
\hline
\end{tabular}

The marginal probability mass function of $N_{2}$ is obtained from Erlang's B formula or (20):

$$
\begin{gathered}
p_{0}^{\prime}=P\left(N_{2}=0\right)=\frac{1}{1+\rho_{2}}, \\
p_{1}^{\prime}=P\left(N_{2}=1\right)=\frac{\rho_{2}}{1+\rho_{2}}, \\
p_{n_{1}, n_{2}}=p_{n_{1}} p_{n_{2}}^{\prime}, \quad n_{1}=0,1,2, \ldots ; n_{2}=0,1 .
\end{gathered}
$$

If (10) and (31) are substituted in (30), the equation (19) is obtained, and if (10) and (32) are substituted in (30), the equation (18) is obtained. Thus, the independence of $N_{1}$ and $N_{2}$ has been demonstrated.

\section{Numerical Results}

The random arrivals and service times were generated from exponential distribution as seconds by using MATLAB 7.10.0 (R2010a) programming for this proposed model. The number of customers taken was 10000 and was performed in three iterations. Performance measures are calculated for different values of $\rho_{i}(i=1,2)$ and three different iterations steps, that is, 100, 1000, and 5000. These results were shown in Tables 1 , 2 , and 3.

\section{Conclusions}

A new queuing discipline is given for a Markov model which consists of two consecutive channels and no waiting line between channels. In this model, steady-state equations, the mean sojourn time, the mean number of customers, and loss probability are obtained. Additionally, two theorems are given which are about optimization of performance measures and the independent of the number of customers, respectively. Performance measures are calculated for different values of $\rho_{i}(i=1,2)$ and for three different iterations steps, that is, 100,1000 , and 5000. Moreover, results of these measures are compared in the tables above. It has been seen that the simulation results approximated the theoretical results. Although the iteration number is increased, the simulation results of performance measures have not changed. However, as $\rho_{i}(i=1,2)$ converges to zero, both the simulation results and exact results approximately are equal to optimal results. Thus, it is said that our proposed queueing model operates well.

For further research, a model, in which a customer who completed his service in channel 1 , blocks channel 1 with probability $\pi$ or leaves the system with probability $1-\pi$ while channel 2 is busy, can be studied.

\section{References}

[1] G. G. O.'Brien, “The solution of some queuing problems," Journal of the Society For Industrial and Applied Mathematics, vol. 2, pp. 133-142, 1954.

[2] R. R. P. Jackson, "Queuing system with phase-type service," Operational Research Quarterly, vol. 5, pp. 109-120, 1954.

[3] P. J. Burke, "The output of a queuing system," Operations Research, vol. 4, pp. 699-704, 1956. 
[4] J. R. Jackson, "Network of waiting lines," Operation Research, vol. 5, pp. 518-524, 1957.

[5] R. B. Cooper, Introduction to Queuing Theory, The Macmillan Company, New York, NY, USA, 1972.

[6] D. Gross and C. M. Harris, Fundamentals of Queueing Theory, John Wiley \& Sons, New York, NY, USA, 3rd edition, 1998.

[7] V. Sağlam and H. Torun, "On optimization of stochastic service system with two heterogeneous channels," International Journal of Applied Mathematics, vol. 17, no. 1, pp. 1-6, 2005.

[8] V. Sağlam and A. Shahbazov, "Minimizing loss probability in queuing systems with heterogeneous servers," Iranian Journal of Science and Technology, vol. 31, no. 2, pp. 199-206, 2007.

[9] A. H. Taha, Operating Research, Macmillian Publishing, New York, NY, USA, 1982.

[10] A. Brandwajn and Y.-L. L. Jow, "An approximation method for tandem queues with blocking," Operations Research, vol. 36, no. 1, pp. 73-83, 1988.

[11] A. A. Akinsete, "Blocked network of tandem queues with withdrawal," Kragujevac Journal of Mathematics, vol. 23, pp. 63-73, 2001.

[12] U. N. Bhat, An Introduction to Queueing Theory, Boston, Mass, USA, 2008.

[13] W. J. Stewart, Probability, Markov Chains, Queues, and Simulation, Princeton University Press, Princeton, NJ, USA, 2009. 


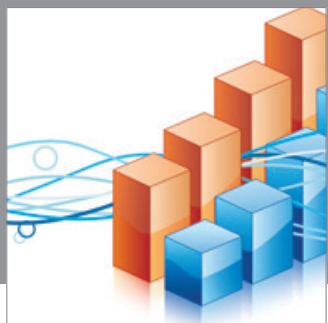

Advances in

Operations Research

mansans

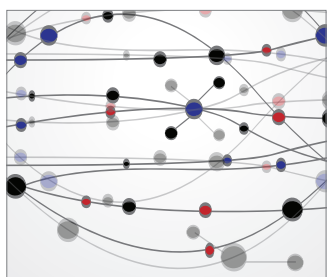

The Scientific World Journal
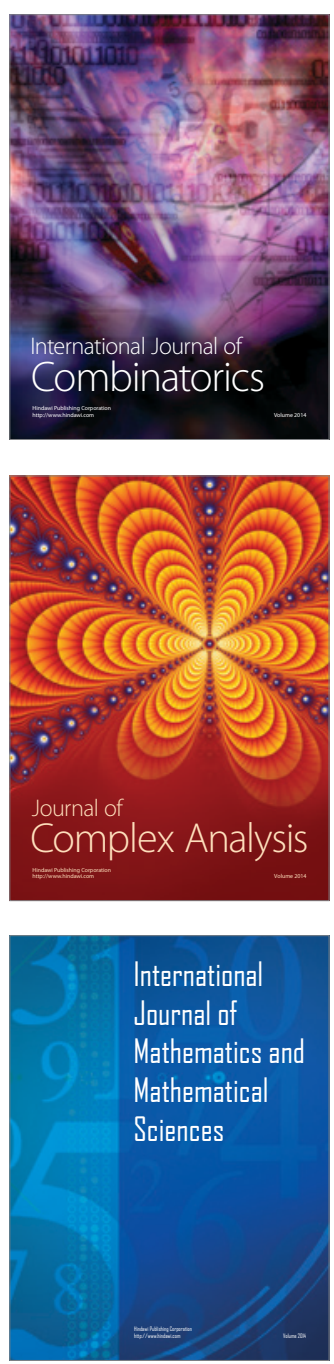
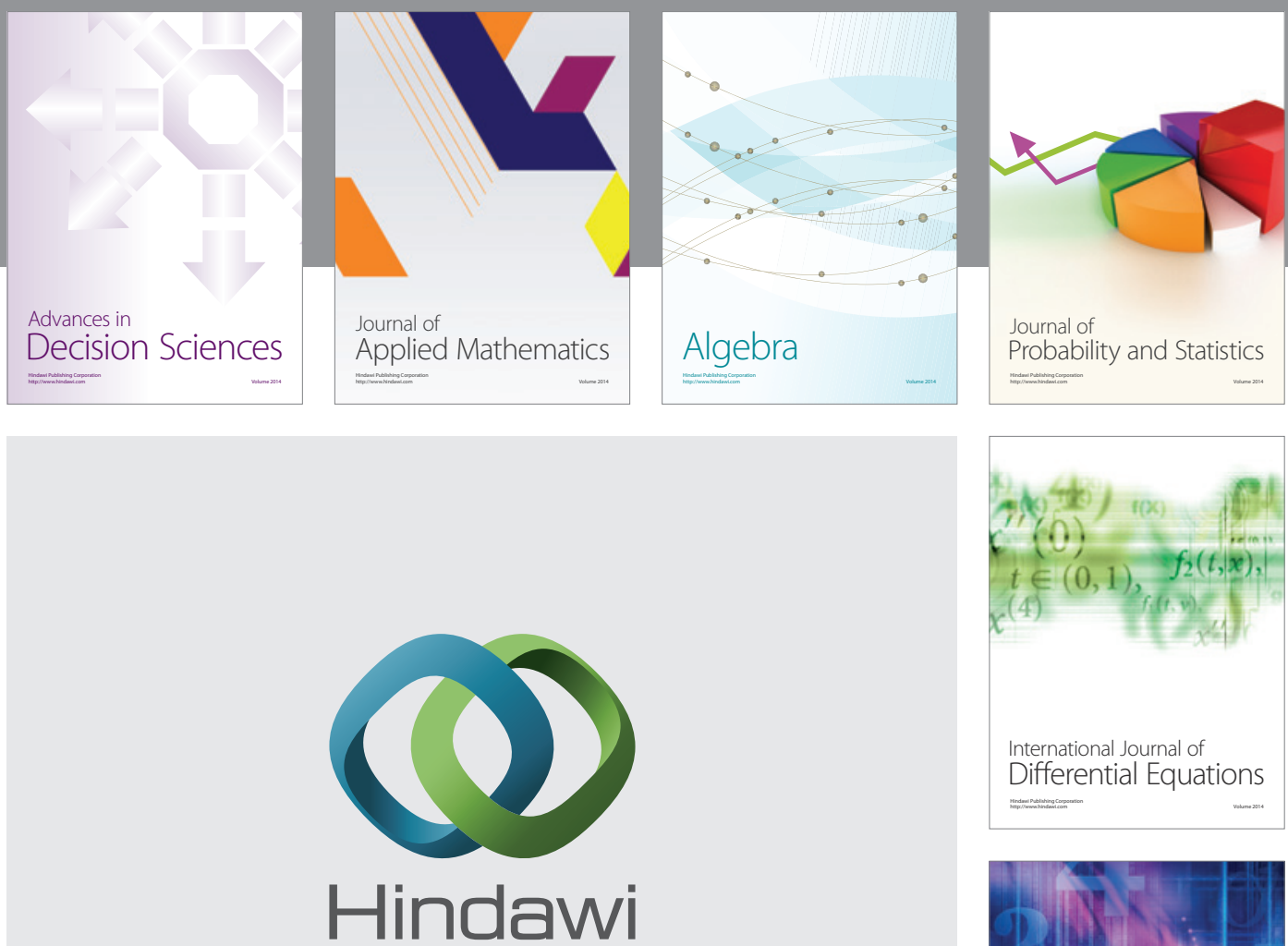

Submit your manuscripts at http://www.hindawi.com
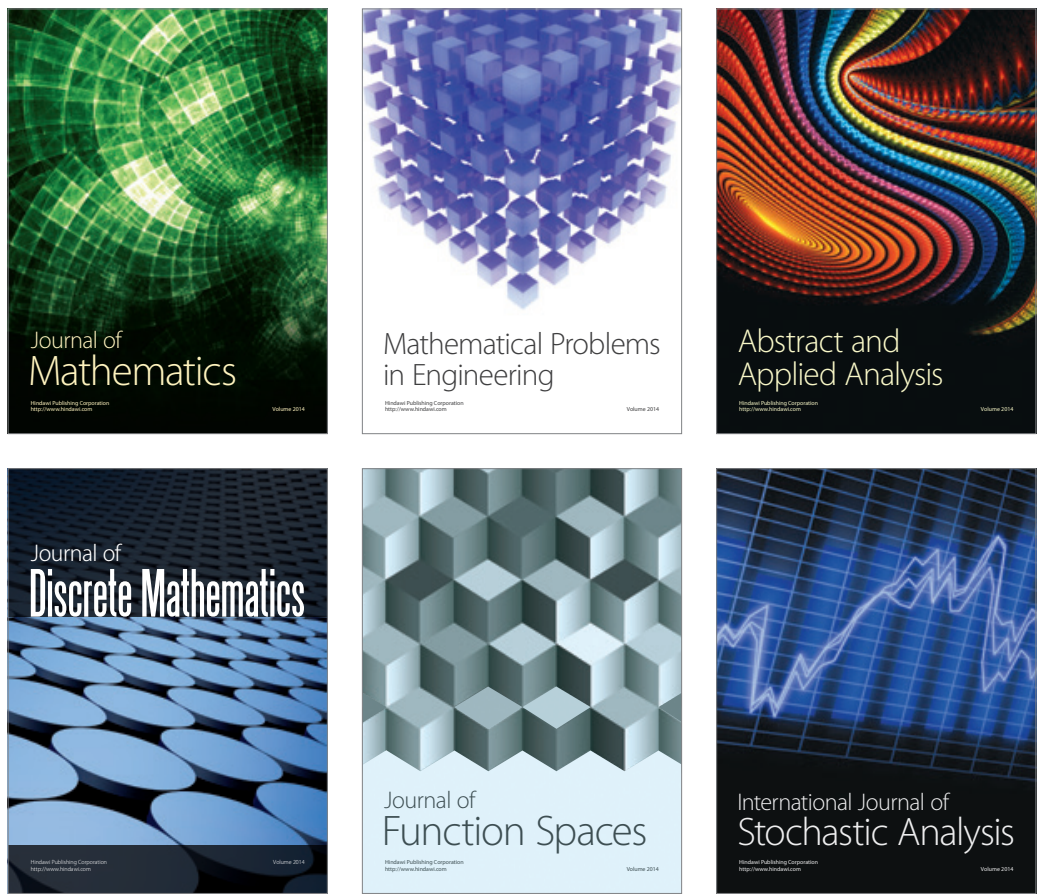

Journal of

Function Spaces

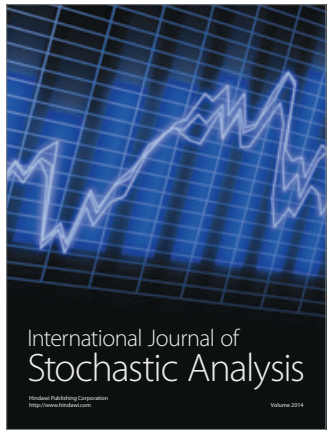

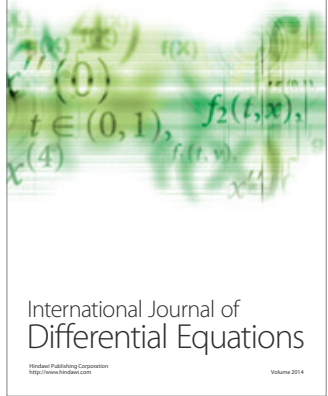
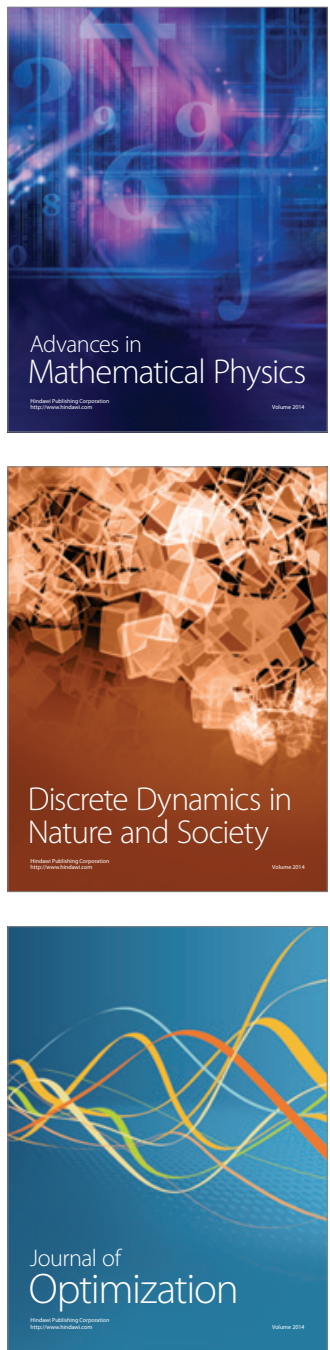